\title{
Tumor Growth Characteristics of the Walker 256 AR Tumor, a Regressive Variant of the Rat Walker 256 A tumor
}

\author{
Fernando Guimarães, Alessandra Soares Schanoski, Tereza Cristina Samico Cavalcanti *, \\ Priscila Bianchi Juliano, Ana Neuza Viera-Matos and Ovídio Rettori \\ Laboratórios Clínicos Especializados; Centro de Atenção Integral à Saúde da Mulher; Universidade de Campinas; \\ C. P.: 6081; 13083-970; Campinas - SP - Brasil
}

\begin{abstract}
The present study aimed at characterizing the subcutaneous development of the Walker 256 (W256) AR tumor, a regressive variant of the rat W256 A tumor. Wistar rats were injected subcutaneously with $4 \times 10^{6}$ W256 A or W256 AR tumor cells. The development of tumors was evaluated daily by percutaneous measurements. None of the W256 A tumors $(n=20)$ regressed, but $62 \%$ of the W256 AR tumor-bearing rats $(n=21)$ underwent complete tumor regression within 35 days. Continuous growth of AR tumors was characterized by an increase of the tumor growth rate from day 12, which reached values above $1.0 \mathrm{~g} /$ day, and were significantly higher $(p<0.05)$ than those of the regressive AR tumors. Immunosuppression by irradiation before subcutaneous injection of AR cells completely abrogated tumor regression and was associated with severe metastatic dissemination. Daily evaluation of the tumor growth rate enabled the discrimination, in advance, between continuously growing tumors and those that regressed later on.
\end{abstract}

Key words: Tumor growth rate; spontaneous tumor regression; Wistar rats; Walker 256

\section{INTRODUCTION}

The Walker 256 (W256) tumor has been extensively used in the studies of cancer pathophysiology, tumor cell metabolism and immune response (Toal et al., 1960; Morrison, 1971; Rettori et al., 1995; Guimarães et al., 1999; Buffon et al., Degasperi et al., 2006; Perroud et al., 2006). Two variants of this tumor have been previously reported as W256 A and B (Guaitani et al., 1982), which are currently known by their growth capacity and lethality after subcutaneous (sc) injection in Wistar rats. This lethality is associated to the severe homeostatic disturbances induced by the W256 tumor, which includes anorexia (Rettori et al., 1995; Guaitani et al., 1982), anemia (Zucker et al., 1977; Vido et al., 2000), thymus atrophy (Rettori et al., 1995) and hydro electrolyte disturbances with glomerular filtration and tubular function alterations (Toal et al., 1960; Morrison, 1971; Rettori et al., 1995; Guimarães et al., 1999; Rettori et al., 2000). Nevertheless, spontaneous W256 tumor regression can occur. This phenomenon is poorly documented (Cavalcanti et al., 2003; Schanoski et al., 2004; Jensen and Muntzing 1970) and has been associated to the rat strain (Schmid et al., 1966; Jaganjac et al., 2008).

We have recently reported the generation of a regressive W256 variant named AR (Schanoski et

*Author for correspondence: samico@caism.unicamp.br 
al., 2004). The AR variant was obtained after serial passages of W256 A tumor cells in the intraperitoneal (ip) cavity of rats, where it grows as an ascitic tumor. All ip serial passage repetitions shifted from A to AR variant within 25 passages. This was characterized by a significant increase in red blood cell osmotic fragility with marked spleen hypertrophy in the host. Besides, MHC class I positive tumor cells significantly increased in the AR variant compared to the W256 A variant (Schanoski et al., 2004).

The objective of the present work was to evaluate the W256 AR variant after sc injection in Wistar rats aiming at a better characterization of the regression phenomenon. The contribution of the immune system for tumor regression was assessed in immune suppressed rats.

\section{MATERIALS AND METHODS}

\begin{abstract}
Animals
Sixty-one male Wistar rats (Rattus novergiccus albinos), at the age of 8-12 weeks were used in the experiments. The rats were housed (5/cage) under controlled temperature $\left(21 \pm 2^{\circ} \mathrm{C}\right)$ and maintained under 12-h light-dark cycle. The animals were fed with standard rat chow (Labina/Purina, Campinas, SP, Brazil) and water, and bedding was autoclaved before use. The general guidelines for animal welfare of the United Kingdom Coordinating Committee on Cancer Research (1988) were followed and all the animal experiments were approved by the Ethical Committee for Animal Experimentation (CEEA-803-1, IB/UNICAMP).
\end{abstract}

\section{Tumor}

Walker 256 tumor cells were originally obtained from the National Cancer Institute Bank, MA, USA. Walker 256 AR variant (W256 AR) was obtained after serial passages of W256 A tumor cells in the ip cavity of rats, as previously described (Schanoski et al., 2004). Both tumor variants are currently kept in the laboratory frozen in liquid nitrogen and maintained through sc (W256 A variant) or ip (W256 A variant and W256 AR variant) passages in rats.

\section{Tumor weight}

The mean percutaneous tumor diameter (average of the two largest transverse diameters) was measured everyday. Tumor weight was estimated from an empirical curve, which related the means of percutaneous diameter and weight. At autopsy, tumors were dissected and weighed, allowing the confirmation of the estimated tumor weight. When necessary, correction of tumor weights recorded along the sc development of the tumors was carried out by introducing a correction factor, as previously described (Rettori et al., 1995).

\section{Tumor growth rate}

Tumor growth rate was calculated according to the formula: (tumor weight in given day - tumor weight 48 h earlier) $/ 2$.

\section{Experiments and animal groups}

Experiment 1 was carried out to compare the sc development of W256 AR and A variants. For this purpose, 21 rats received a single sc injection of $4 \times 10^{6} \mathrm{~W} 256$ AR cells, and 20 rats received a single sc injection of $4 \times 10^{6}$ W256 A cells. Both W256 tumor cell suspensions were prepared in Ringer solution at $20 \times 10^{6}$ viable tumor cells $/ \mathrm{ml}$ and injected in the dorso-lumbar region of the rats. Experiment 2 assessed the involvement of the immune system in the W256 AR tumor development and regression. For this purpose, 10 rats were immunesuppressed by total body irradiation (4 Gy) (Anderson and Warner, 1976), and injected with tumor cells after a 5-day recovering period, in which food intake approached normality. Irradiated (irr) rats received a single sc injection of $4 \times 10^{6} \mathrm{~W} 256 \mathrm{AR}$ cells. The control group, containing 10 non-irradiated (nonirr) rats, was equally injected in the sc with $4 \times 10^{6}$ W256 AR cells of the same tumor cell suspension. The W256 AR cell suspension was prepared in Ringer solution at $20 \times 10^{6}$ viable tumor cells $/ \mathrm{ml}$ and, as usual, injected in the dorso-lumbar region of the rats.

\section{Autopsy}

Autopsies were performed on all tumor-bearing rats, when the tumors were dissected and weighed. Rat body weight was assessed after tumor dissection. During autopsies, special attention was given to the presence of metastasis, invasion of important tissue and bleeding.

\section{Statistics}

The results were reported as mean \pm standard error of the mean (SEM), and differences between the W256 AR regression and continuous tumor 
growth were determined by the Wilcoxon test ( $\mathrm{P}<$ 0.05) (Snedecor and Cocchran, 1989).

\section{RESULTS}

\section{Experiment 1}

All sc injections with W256 A and AR tumor cell variants resulted in tumor growth. Tumors were detected after 3-6 days, teeny nodules, not adhered to the muscle tissue, could be noticed by palpation growing in the site of injections. Tumor diameters started being recorded short after their first detection when tumor sizes achieved $8 \mathrm{~mm}(0,05 \mathrm{~g})$ and, therefore, allowing the percutaneous measuring. Figure 1 presents tumor weight curves obtained from rats injected with W256 AR $(n=21)$ variant as individual data, whereas the tumor weight curve from rats injected with W256 A $(n=20)$ variant is presented as average. As expected, the W256 A tumors developed very similarly among the tumor-bearing rats and regressions were not observed. Contrary to this, the development of W256 AR tumors was highly variable, resulting in continuous growth $(5$ out of 21 rats, $24 \%$ ) or complete regression (13 out of 21 rats, $62 \%)$.

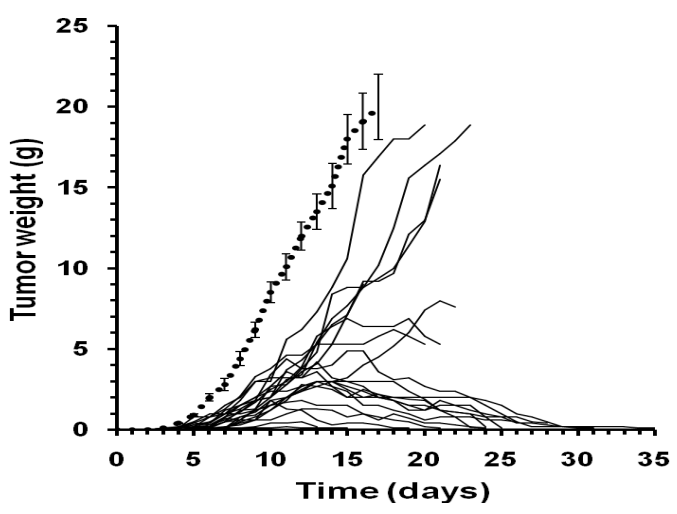

Figure 1 - Tumor weight curves comparing tumor development of the W256 A and AR variants. Solid lines: individual data obtained from 21 rats receiving sc injection of $4 \times 10^{6}$ cells of the W256 AR variant; Doted line: average calculated from 20 rats receiving sc injection of $4 \times 10^{6}$ cells of the W256 A variant. Data are reported as mean \pm SEM.

Three AR tumor-bearing rats were sacrificed (around the $22^{\text {nd }}$ day after tumor cell injection) to comply with an experimental protocol (not shown here), establishing tissue sampling just before the tumor regression (indicated by moderate tumor development followed by its stabilization). The W256 AR tumors peaked at variable weights (from less than 1 to $8 \mathrm{~g}$ ) and at variable times (10 to 21 days) before regression. Tumor regressions were completed within 35 days after sc injection $(\mathrm{n}=13)$.

\section{Experiment 2}

All sc injections with W256 AR tumor cells carried out in irr and non-irr rats resulted in tumor growth. Similar to the experiment 1 , tumors were detected 3-5 days following sc injections, when teeny nodules, not adhered to the muscle tissue, could be noticed by palpation. Figure 2 presents tumor weight curves for the W256 AR variant obtained from non-irr $(\mathrm{n}=10)$ and irr $(\mathrm{n}=10)$ tumor bearing-rats. Tumor regressions were observed in all the non-irr rats (100\% of the animals). However, treating the rats with total body irradiation before tumor cell injections abrogated completely W256 AR tumor regression. In addition to the continuous tumor growth, the irrrats quickly developed the systemic effects (not shown here) induced by Walker tumors, causing the premature sacrifice of the tumor-bearing irrrats.

\section{Sc tumor growth rates}

Figure 3 presents tumor growth rates obtained from both W256 A and AR variants during the experiments 1 and 2. In Experiment 1, W256 AR tumor growth rates were calculated by assembling tumor-bearing rats in two groups: one presenting tumor regression $(n=13)$ and the other presenting continuous tumor growth $(n=5)$. 


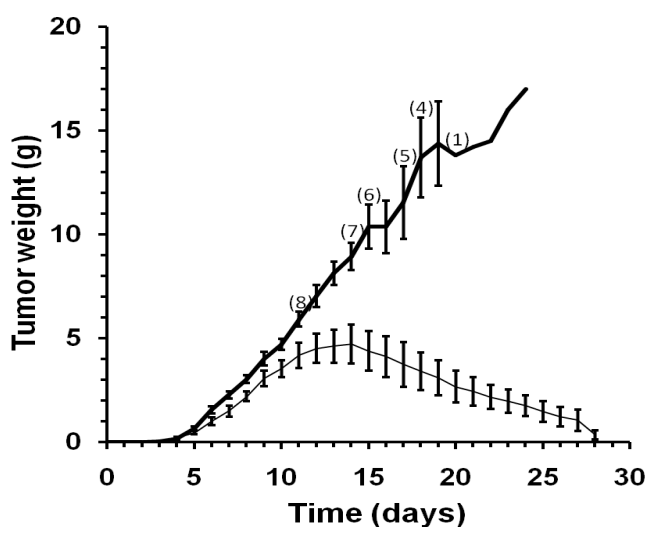

Figure 2 - Differential effect of immunosupression on tumor growth in rats receiving sc injection of $4 \times 10^{6}$ cells of the W256 AR variant. Thin line: average tumor weight curve of 10 non-irr rats; Thick line: average tumor weight curve of 10 irr rats. Data are reported as mean \pm SEM. Numbers within parentheses indicate the remaining number of animals at that moment.

The continuous growth of W256 AR tumors was characterized by an increase of tumor growth rate from approximately $0.7 \mathrm{~g} /$ day (day $12,0.74 \pm 0.24$ g/day, Figure 3, Experiment 1) to more than 1.0 g/day (day $14,1.43 \pm 0.27 \mathrm{~g} /$ day). This tumor growth increase was significantly higher $(\mathrm{p}<0.05)$ than the one observed for regressive AR tumors (day 14, $0.10 \pm 0.10 \mathrm{~g}$ /day) and, therefore, approaching tumor growth rate characteristic of the W256 A variant (1.50-2.00 g/day). W256 AR tumor regressions were preceded by moderate tumor growth rates around $0.4 \mathrm{~g} /$ day, (day 12, 0.37 \pm 0.09 g/day, Figure 3, Experiment 1), which gradually diminished from day 12-14 until negative values, indicating tumor regression. W256 AR tumor-bearing rats sacrificed on day 21$22(\mathrm{n}=3)$ presented tumor growth rates consistent to regressive tumors.
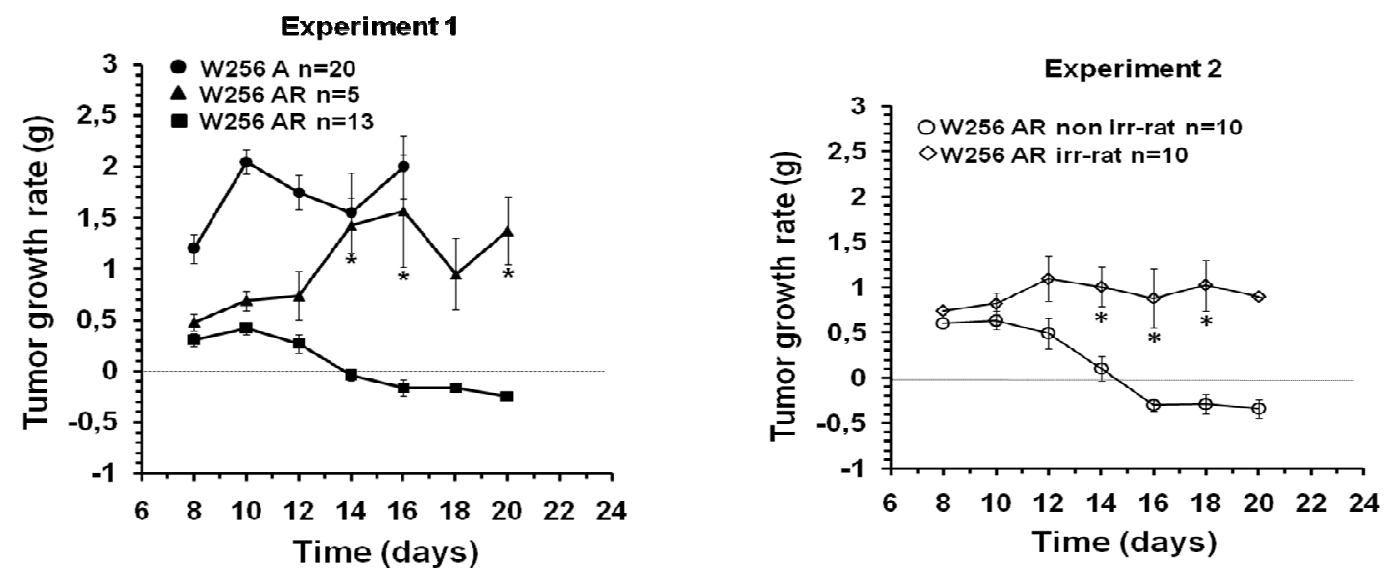

Figure 3 - Experiment 1: Differential tumor growth rates in rats receiving sc injection of $4 \times 10^{6}$ cells of the W256 A variant (black circle, $n=20$ ) or $4 \times 10^{6}$ cells of the W256 AR variant (black triangle, $n=5$ continuous tumor growth; black square, $n=13$ spontaneous tumor regression). Experiment 2: Effect of immunosupression on the W256 AR tumor development. Differential tumor growth rates in 10 non-irr rats (circules) and 10 irr rats (squares) receiving $4 \times 10^{6}$ cells of the W256 AR variant. Data are reported as mean \pm SEM. *statistically significant differences between AR tumors growing continuously and AR tumors regressing $(\mathrm{P}<0.05$ Wilcoxon test). 
Non-irr rats of the Experiment 2 reproduced the same tumor regression pattern for W256 AR tumors as observed during experiment 1 (Figure 3). However, the rat pretreatment of total body irradiation previously to the W256 AR cell injections resulted in continuous tumor growth as mentioned above and in the increase of tumor growth rates to about $1.0 \mathrm{~g} / \mathrm{day}$. The increase of tumor growth rates in irr rats took place around day 12 after sc injection of tumor cells (Figure 3, Experiment 2). This result was similar to the nonwrr rats presenting continuous tumor growth.

\section{Autopsies}

Tumors dissected during autopsies were found adhered to the dorsal muscle adjacent to the site of the tumor cell injection. Macroscopic alterations (small nodules 3-4 $\mathrm{mm}$ of diameter) identified as metastases were found in $75 \%$ of the rats injected with W256 A variant and in $11 \%$ of the rats injected with W256 AR variant, always restricted to the lymph nodes (inguinal, axillary and paraaortic lymph nodes). On the other hand, autopsies revealed a severe metastatic dissemination in all irr-rats $(100 \%)$. Metastases were found not only in lymph nodes of irr-rats, but were also spotted over the muscle wall of the thorax and abdomen, and spread in internal organs such as the gut and lungs (Figure 4).

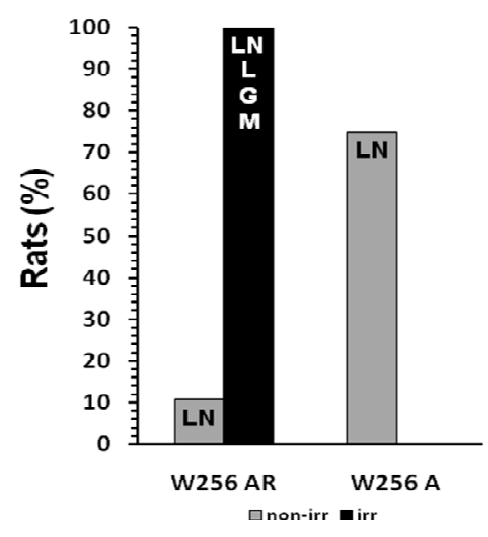

Figure 4 - Metastatic dissemination of W256 A and AR variants (non-irr rats = gray bars; and irr rats = black bar). Metastases were found in lymph nodes (LN) of irr-rats, spotted over the muscle wall $(\mathrm{M})$ of the thorax and abdomen, and spread in internal organs such as the gut $(\mathrm{G})$ and lungs $(\mathrm{L})$.

\section{DISCUSSION}

\section{W256 tumor regression}

It is currently well known that W256 tumor is characterized by its continuing growth capacity. This characteristic has been widely reported since Dr. Walker first succeeded in maintaining this tumor by sc passages in rats. According to Earle (1935), the W256 tumor was obtained after serial sc transplants from a tumor that had originally appeared spontaneously in an adult female rat. Earle reported that after several serial tumor sc transplants from the original donor to groups of young receptor rats, it was possible to finally obtain one hundred percent tumor growth at each sc passage. That high percentage of tumor growth was observed even when different rat strains were used. During all the transfers in the laboratory from 1928 to 1932 , Dr. Walker found only 4 instances of regression of the tumor.

Spontaneous regressions of W256 are still rarely reported. Several reasons account for such low frequency, including that most of the studies using the W256 tumor model exploits its growth capacity and ability to induce severe systemic alterations in the host. Consequently, a regressive variant of W256 tumor would not be suitable for studying these goals. Eventually, W256 tumor regressions might have been overlooked since tumor-bearing rats could be sacrificed prior to when the tumor regression took place, in order to comply with the necessity of sacrificing rats with large tumors. 
Few reports on spontaneous regressions of the W256 tumor have mainly connected this phenomenon to the rat strain, such as SpragueDawley rats, Buffalo rats and Fischer rats (Schmid et al., 1966; Jaganjac et al., 2008). In the present study, high amounts of spontaneous tumor regressions were observed even in Wistar rats. However, they were connected to the AR variant of the W256 tumor. In total, $62 \%$ of the W256 AR tumor-bearing rats presented complete tumor regression, but none of the Walker 256 A tumors regressed. Regardless of the low number of references reporting spontaneous regression of W256 tumor, it was possible to establish a regressive tumor variant, named $\mathrm{AR}$, which was originated by serial passages of the W256 A tumor cells in the ip cavity of Wistar rats (Schanoski et al., 2004). The shift on the characteristics of W256 tumor, from a progressive variant to an immunogenic and regressive variant by serial ip passages of the tumor cells, could also explain the spontaneous regressions of the W256 tumors reported by Jensen and Müntzing (1970).

\section{W256 AR tumor in immunosuppressed rats}

The involvement of the immune system in W256 AR tumor regression was assessed in the rats immunosuppressed by irradiation. Rats subjected to total body irradiation (4 Gy) presented $80 \%$ reduction of circulating lymphocytes (from $7.5 \times 10^{3} / \mu 1$ to $\left.1-2 \times 10^{3} / \mu 1\right)$. Low numbers of circulating lymphocytes remained for approximately two weeks after the irradiation (data not shown), impairing the development of a proper adaptive immune response which has been accounted for tumor regressions (Pardoll and Topalian, 1998; Rees and Mian, 1999). W256AR regressions were completely abrogated in irr-rats. Besides the continuous growth of the tumors, irrrats also presented a marked metastatic dissemination. On the other hand, non-irr rats presented $100 \%$ of tumor regression, followed by the development of immunity against both $\mathrm{A}$ and AR variants. Immunization was confirmed by randomly challenging the rats with high-dose injections of W256 tumor cells (data not shown). The results confirmed the AR variant as an immunogenic variant of the W256 tumor. The improved immunogenicity of W256 AR variant was also consistent to previous studies showing that W256 AR variant presented higher numbers of MHC class I positive cells than W256 A variant (Schanoski et al., 2004).

\section{Tumor growth rate and outcome of W256 AR tumors}

The sc development of the W256 AR tumors was highly variable when compared to the W256 A variant, since AR tumors could reach variable weights at variable times before regression. Besides, different W256 AR tumor cell suspensions could result from 60 to $100 \%$ of tumor regressions after single sc injection in the rats. In spite of this variability, the data presented here showed that the outcome of the W256 AR tumors (regression or continuous growth) could be predicted when tumor growth in the rate was taken into consideration. Continuous growth of W256 AR tumors was associated with the high tumor growth rates, approximately $1.0 \mathrm{~g} /$ day or higher, beginning from day 12-14 after sc injection of W256 AR cell variant. Conversely, AR tumor regressions were associated with lower tumor growth rates (about $0.5 \mathrm{~g} /$ day) that gradually slowed down from day 12-14 after sc injections. Tumor growth rate provides a useful parameter for the experimental animal evaluation, particularly within the period preceding tumor regression. Therefore, tumor growth rate helps to make decision regarding the sacrifice of experimental animals, since the rats presenting regressive tumors (low tumor growth rate) can be kept longer than the rats presenting progressive ones (high tumor growth rate). Besides, by taken tumor growth rate into consideration, important host alterations, mainly related to the immune system, can be clearly observed, giving the possibility of separating the individuals with progressive tumors from those with regressive tumors before averaging the data.

Interestingly, the fact that variation in the tumor growth rates took place from day 12-14 might indicate the involvement of adaptive immune response. Thus, the decrease in AR tumor growth rates possibly indicate a successful adaptive immune response, whereas increase of tumor growth rate would indicate the opposite. Favorable to this explanation was the fact that irr rats failed in promoting AR tumor regression, which was associated with the increased tumor growth rate from day 12-14. Additionally, W256 AR tumor regressions are associated with normal $\mathrm{CD} 4^{+} / \mathrm{CD} 8^{+}$ ratio, whereas tumor continuous growth is associated with a significant reduction in the $\mathrm{CD}^{+} / \mathrm{CD}^{+}$ratio (Guimarães, 2000). W256 AR tumor-bearing rats also present plasma compounds resembling immunoglobulin, around the same 
period (day 12-14) when tumor regressions started (Cavalcanti et al., 2003).

The precise mechanism underlying the origin of W256 AR variant is unknown. Also, unknown are the mechanisms that promote the shift from AR to A variant by serial sc passages of fragments, taken from the tumors of high growth rate (personal observation). Nevertheless, it was demonstrated that W256 solid tumors consisted of both MHC class I positive and negative cells (Schanoski et al., 2004). Additionally, AR variant comprise higher frequency of MHC class I positive cells than the A variant. It is widely accepted that new tumor variants result from differential propagation of tumor subclones in their microenvironment in a process that includes the immune system (Khong and Restifo, 2002). Currently, the cancerimmunoediting hypothesis offers a theoretical framework including immune cells, antibodies, cytokines and chemokines to explain how interactions between the emerging tumors and immune system would result in tumor elimination or even in tumor outgrowth, by sculpting tumor immunogenicity or by inhibiting the hostprotective antitumor responses (Dunn et al., 2002; Dunn et al., 2006; Bui and Schreiber, 2007). In this context, it could be suggested that the shift from W256 A to AR variant into the peritoneal cavity might be a consequence of the immunoselection of $\mathrm{MCH}$ class I negative cells associated with the induction of MHC class I expression on tumor cells by INF- $\gamma$ (Schanoski et al., 2004; Malmberg et al., 2002). The present study successfully characterized the sc development of W256 AR variant, emphasizing that spontaneous tumor regression could be observed even in Wistar rats. Daily evaluation of the tumor growth rate enables discrimination of the W256 AR tumors presenting continuous growth from the regressive tumors. Abrogation of tumor regressions in immunosuppressed rats suggests the immune system as effector of W256 AR tumor regression. The data described in the present work would be essential for the correct handling of the Walker 256 tumor cells, which could be useful as a tumor model for the study of tumor immunoediting mechanisms.

\section{ACKNOWLEDGEMENTS}

Alessandra Soares Schanoski was recipient of a CAPES fellowship (2067-030). This research was supported by PROAP/CAPES and CAISM/UNICAMP. The authors are grateful to Amilton Garcia for animal care.

\section{RESUMO}

O objetivo neste estudo foi caracterizar o desenvolvimento subcutâneo do tumor de Walker 256 (W256) AR, uma variante regressiva do tumor de W256 A de rato. Ratos Wistar foram injetados com $4 \times 10^{6}$ células tumorais de W256 A ou W256 AR. O desenvolvimento tumoral foi avaliado diariamente. Nenhum dos tumores W256 A $(n=20)$ regrediu, mas $62 \%$ dos ratos com tumor W256 AR apresentaram regressão completa dos tumores em até 35 dias. $\mathrm{O}$ crescimento contínuo dos tumores AR foi caracterizado pelo aumento da taxa de crescimento tumoral a partir do dia 12, alcançando valores maiores que $1,0 \mathrm{~g} / \mathrm{dia}$, que foram significativamente superiores $(\mathrm{p}<0,05)$ aos valores de taxa de crescimento dos tumores regressivos AR. A imunossupressão por irradiação precedendo a injeção das células tumorais $\mathrm{AR}$ eliminou completamente a regressão tumoral e favoreceu disseminação metastática severa. Este estudo caracterizou o desenvolvimento do tumor de W256 AR em condições específicas, documentando a regressão espontânea deste tumor após a injeção subcutânea de altas doses de células tumorais em ratos Wistar. A avaliação diária da taxa de crescimento tumoral permite discriminar precocemente os tumores com crescimento continuo daqueles que são regressivos. A taxa de crescimento tumoral é um parâmetro útil para a avaliação dos animais experimentais, particularmente no período que precede a regressão dos tumores.

\section{REFERENCES}

Anderson, R.E.; Warner, N.L. (1976), Ionizing radiation and the immune response. Adv. Immunol, 24, 215-35.

Buffon, A.; Ribeirom, V.B.; Schanoski, A.S.; Sarkis, J.J.F. (2006), Diminution in adenine nucleotide hydrolysis by platelets and serum from rats submitted to Walker 256 tumour. Mol Cell Biochem, 281, 18995.

Bui, J.D.; Schreiber, R.D. (2007), Cancer immunosurveillance, immunoediting and inflammation: independent or interdependent processes? Curr Opinion Immunol, 19, 203-8. 
Cavalcanti, T.C.; Gregorini, C.C.; Guimarães, F.; Rettori, O.; Vieira-Matos, A.N. (2003), Changes in red Blood cell osmotic fragility induced by total plasma and fractions from rats bearing progressive and regressive variants of the Walker 256 tumor. Braz J Med Biol Res, 36, 887-95.

Degasperi, G.R.; Zecchin, K.G.; Borecký, J.; CruzHöfling, M.A.; Castilho, R.F.; Velloso, L.A.; Guimarães, F.; Vercesi, A.E. (2006), Verapamilsensitive $\mathrm{Ca} 2+$ channel regulation of Th1-type proliferation of splenic lymphocytes induced by Walker 256 tumor development in rats. Eur $J$ Pharmacol, 549, 179-84.

Dunn, G.D.; Koebel, C.A.; Schreiber, R.D. (2006), Interferons immunity and cancer immunoediting. Nat immunol, 6, 836-48.

Dunn, G.P.; Bruce, A.T.; Lloyd, J.O.; Schreiber, R.D. (2002), Cancer immunoediting: from immunosurveillance to tumor scape. Nat Immunol, 3, 991998.

Earle, W.R. (1935), A study of the Walker rat mammary carcinoma 256, in vivo and in vitro. Am. J. Cancer, 24, 566-612.

Guaitani, A.; Recchia, M.; Carli, M.; Rocchtti, M.; Bartosek, I.; Garattini, S. (1982), Walker carcinoma 256: a model for studies on tumor induced anorexia and cachexia. Oncology, 39, 173-8.

Guimarães, F.; Rettori, O.; Fernandes, G.A. (1999), The influence of septal lesions on sodium and water retention induced by Walker 256 tumor. Braz J Med Biol Res, 32, 309-17.

Guimarães, F. (2000), Influência da lesão da área septal do cérebro de ratos sobre os linfócitos T (CD4+ e $\mathrm{CD} 8+$ ) do baço e efeitos sistêmicos induzidos pelo tumor de Walker 256. PhD Thesis, Universidade Estadual de Campinas, Campinas, Brazil.

Jaganjac, M.; Poljak-Blazi, M.; Zarkovic, K.; Schaur, R.J.; Zarkovic, N. (2008), The involvement of granulocytes in spontaneous regression of Walker 256 carcinoma. Cancer Lett, 260, 180-86.

Jensen, G.; Muntzing, J. (1970), Differences in the growth of the Walker carcinoma in Sprague-Dawley and Wistar rats. Z. Krebsforsch, 74, 55-8.

Khong, H.T.; Restifo, N.P. (2002), Natural selection of tumor variants in the generation of "tumor escape" phenotypes. Nat Immunol, 3, 999-1005.

Malmberg, K.J.; Levitsky, V.; Norell, H.; de Matos, C.T.; Carlsten, M.; Schedvins, K.; Rabbani, H.; Moretta, A.; Söderström, K.; Levitskaya, J.; Kiessling, R. (2002), IFN-gamma protects short-term ovarian carcinoma cell lines from CTL lysis via a CD94/NKG2A-dependent mechanism. J Clin Invest, 110, 1515-23.

Morrison, S.B. (1971), Water intake and exchange and hydration of rats during growth of Walker 256 carcinosarcoma. J Natl Cancer Inst, 46, 825-30.
Pardoll, D.M.; Topalian, S.L. (1998), The role of CD4+T cell responses in antitumor immunity. Curr Opinion Immunol, 10, 588-94.

Perroud, A.P.A.S.; Ashimine, R.; Castro, R.M.; Guimarães, F.; Vieira, K.P.; Vilella, C.A.; Cavalcanti, T.C.S.; Zollner, R.L. (2006), Cytokine gene expression in Walker 256: A comparison of variants A (aggressive) and AR (regressive). Cytokine, 36, 123-33.

Rees, R.C.; Mian, S. (1999), Selective MHC expression in tumours modulates adaptative and innate antitumour responses. Cancer Immunol Immun, 48, 374-81.

Rettori, O.; Vieira-Matos, A.N.; Gontijo, J.A.R. (2000), Re-assessment of the renal hydrosaline dysfunction in rats bearing the Walker tumor. Renal Failure, 22, 769-84.

Rettori, O.; Vieira-Matos, A.N.; Tahin, Q.S. (1995), Variability and discontinuity of the pathognomonic systemic effects caused by the Walker 256 tumor progression in rats. Tumori, 81, 370-7.

Schanoski, A.S.; Cavalcanti, T.C.; Campos, C.B.L.; Viera-Matos, A.N.; Rettori, O.; Guimarães, F. (2004), Walker 256 tumor MHC class I expression during the shift from A variant to the immunogenic AR variant. Cancer Lett, 211, 119-27.

Schmid, F.A.; Cappuccino, J.G.; Merker, P.C.; Tamowski, C.S.; Stock, C.C. (1966), Chemoterapy Studies in an animal spectrum. I. Biological characteristics of the tumors. Cancer Res, 26, 172-80.

Snedecor, G.W.; Cocchran, W.G. (1989), Shortcut and non parametric methods. In-Statistical Methods, ed. G.W. Snedecor, W.G. Cocchran. State University Press, Ames, Iowa, pp 71-2, 138-40.

Toal, J.N.; Millar, F.K.; Brooks, R.H.; White, J. (1960), Sodium retention by rats bearing the Walker carcinosarcoma 256. Am J Phys, 200, 175-81.

United Kingdom Coordinating Committee on Cancer Research (1988), Guidelines for welfare of animals in experimental neoplasia. Lab Anim, 22, 195-201.

Vido, A.A.; Cavalcanti, T.C.; Guimarães, F.; VieiraMatos, A.N.; Rettori, O. (2000), The hemolytic component of cancer anemia: effects of osmotic and metabolic stress on the erythrocytes of rats bearing multifocal inoculations of the Walker 256 tumor. Braz J Med Biol Res, 33, 815-22.

Zucker, S.; Lysic, R.M.; Di Stefano, J. (1977), Pathogenesis of anemia in rats with Walker 256 carcinosarcoma. J Clin Med, 90, 502-11.

Received: August 18, 2008; Revised: March 26, 2009; Accepted: April 14, 2010. 